\title{
Novel Case of Emergency Room to Operation Theatre for Management of Blunt Chest Wall Injury
}

\author{
Kelsie B Crathern, Don G Campbell, Martin E Wullschleger and Bhavik M Patel*
}

Division of Trauma, Gold Coast University Hospital, Southport, Queensland, Australia

${ }^{\star}$ Corresponding author: Bhavik Patel, Division of Trauma, Gold Coast University Hospital, Southport, Queensland, Australia, Email: drbhavikpatel@hotmail.com

Received: April 17, 2020; Accepted: April 23, 2020; Published: April 27, 2020

We here by present a novel case of Emergency Room to Operation Theatre for management of Blunt Chest Wall Injury. This is the first case to our knowledge in Australasian literature highlighting the importance of transfer from the Emergency Room to Operative room for early correction of respiratory physiology. A 74-year -old patient was air lifted into our level I trauma centre from a peripheral hospital. This was following fall from 3-meter height whilst on a ladder. On clinical examination the patient was able to communicate and maintaining their own airway with oxygen requirement of 15 litres via a non- breather mask to achieve oxygen saturation of $92 \%$.

On examination, there was extensive subcutaneous emphysema and limited movement of the left chest wall on both inspiration and expiration. Prior to being air lifted, a left sided intercostal catheter placed by the peripheral hospital which was swinging with minimal amount of hemoserous drainage. The hemodynamics suggested a pulse rate was 115- sinus rhythm and non -supported blood pressure of $122 / 80 \mathrm{~mm}$ of $\mathrm{Hg}$. Venous blood gas results were as follows- $\mathrm{pH}$ 7.16, p CO2 - 70, p O2- 28 and base excess of -5.2. A trauma series scan at the peripheral hospital was suggestive of isolated chest wall injury with anterolateral bi cortical displaced rib fractures and moderate amount of hemopneumothorax (Figure 1A).

We organised a repeat chest $\mathrm{x}$-ray (Figure 1B). The $\mathrm{x}$-ray confirmed multi-level rib fractures with associated pneumothorax.

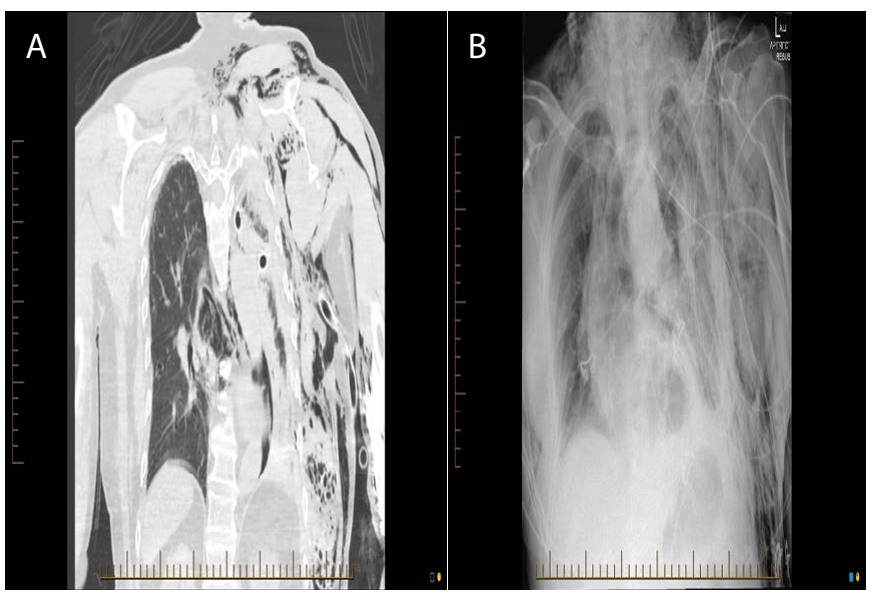

Figure 1: (A) Computed Tomography of the chest with extensive subcutaneous emphysema and bi cortical displaced rib fractures.

(B): X-Ray chest on presentation.

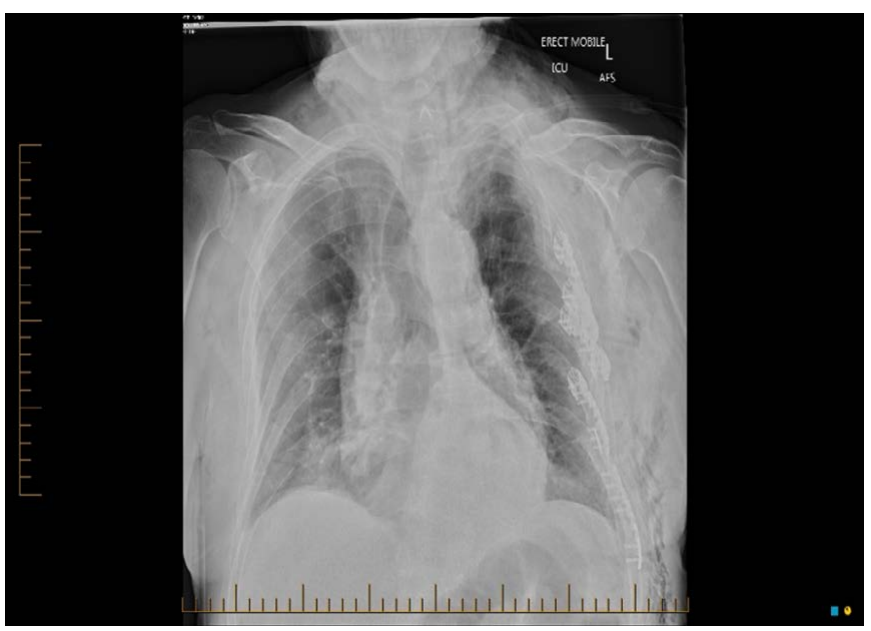

Figure 2: Post-Operative X-ray chest.

Patient was in respiratory failure with a blood carbon dioxide level of 70 and oxygen of 28 , thus we decided to procced with surgical stabilisation of rib fractures directly from the emergency department. General anaesthesia was administered via a single lumen tube. Right lateral position with an incision parallel to the lateral border of scapula was undertaken. Open reduction and internal fixation of rib numbers 3,4,5,6,7 was achieved using Rib Loc (8055 NE Jacobson St., Suite 700 Hillsboro, OR 97124). Ongoing arterial blood gases whilst on theatre table as the stabilisation progressed suggested a fall in CO2 levels to 35 . Figure 2 depicts the post- operative $x$-ray. Patient was extubated the following day and was discharged home on day 5 .

\section{Discussion}

This is the first case to our knowledge in Australasian literature highlighting the importance of transfer from the Emergency Room to Operative room for early correction of respiratory physiology. Elderly patients who sustain blunt chest trauma with rib fractures have twice the mortality and thoracic morbidity of younger patients with similar injuries. For each additional rib fracture in the elderly, mortality increases by $19 \%$ and the risk of pneumonia by $27 \%$ [1]. Literature review suggests in adult patients with flail chest, surgical stabilisation of rib fractures decrease mortality; shorten duration of mechanical ventilation, hospital length of stay, and Intensive Care Unit length of stay; and decrease incidence of pneumonia and need for tracheostomy [2]. 
The goal at our Level I trauma centre is to attempt stabilisation of the chest wall as soon as hemodynamic stability is established. This case highlights the importance of early surgery to avoid potential morbidity and mortality for adult patients with blunt chest wall injury.

\section{References}

1. Bulger EM, Arneson MA, Charles N. Gregory MJ, Jurkovich (2000) Rib fractures in the elderly. The Journal of Trauma: Injury, Infection, and Critical Care 48: 1040-1047.

2. Kasotakis, Hasenboehler EA, Streib EW, Patel N, Patel MB, et al., (2017) Operative fixation of rib fractures after blunt trauma: A practice management guideline from the Eastern Association for the Surgery of Trauma. Journal of Trauma and Acute Care Surgery 82: 618-626.

\section{Citation:}

Kelsie B Crathern, Don G Campbell, Martin E Wullschleger and Bhavik M Patel (2020) Novel case of Emergency Room to Operation Theatre for management of Blunt Chest Wall Injury. Integr J Orthop Traumatol Volume 3(1): 1-2. 